severe instability and needed both adrenaline and noradrenaline. Fourteen (54\%) of 26 children had metabolic acidosis, and more than $30 \%$ patients had electrolyte abnormality of which hypocalcemia was the commonest followed by hypokalemia. Average amount of PRBC transfusion was 40.7 $\pm 26.5 \mathrm{~mL} / \mathrm{kg}$; WBC $25.7 \pm 12.7 \mathrm{~mL} / \mathrm{kg} ;$ FFP $20.5 \pm 12.8 \mathrm{~mL} /$ $\mathrm{kg}$; platelets $7.7 \pm 4.05 \mathrm{~mL} / \mathrm{kg}$; and cryo $4.77 \pm 2.32 \mathrm{~mL} / \mathrm{kg}$. 3 patients $(22.5 \%)$ had mild transfusion reaction and needed antihistamine and steroids. Postoperative parameters: Out of 26 patients, 11 (42.3\%) were electively ventilated for average of 23 hours. $6 / 26$ patients (23.1\%) were on inotropic support for 10 to 21 hours. Average days of ICU and hospital stay were 1.5 and 10 days, respectively. 11/26 had postoperative complication. There was no death reported in this series.

Conclusions: Anticipation, preparation, and aggressive intraoperative resuscitation with blood and blood products during massive blood loss reduce the postoperative morbidity and mortality.

\section{A0022 Effect of Remote Ischemic Preconditioning on Cerebral Vasospasm and Biomarkers of Cerebral Ischemia in Aneurysmal Subarachnoid Hemorrhage}

Sangeetha R. Palaniswamy, ${ }^{1}$ Venkatapura J. Ramesh, ${ }^{1}$ Sriganesh, ${ }^{1}$ Rita Christopher, ${ }^{1}$ Dhananjaya Bhat ${ }^{2}$

${ }^{1}$ Department of Anaesthesia, National Institute of Mental

Health and Neurosciences, Bengaluru, India

${ }^{2}$ Department of Neurosurgery, National Institute of Mental Health and Neurosciences, Bengaluru, India

Background: Cerebral vasospasm is a dreaded complication of aneurysmal subarachnoid hemorrhage (aSAH) predisposing one to delayed cerebral ischemia. We investigated the cerebroprotective effects of remote ischemic preconditioning (RIPC) in patients with aSAH.

Materials and Methods: This was a single-center, prospective, parallel-group, randomized, pilot trial, approved by institutional ethics committee. Patients with aSAH admitted to National Institute of Mental Health and Neurosciences for surgical clipping, fulfilling the trial inclusion criteria, were randomized to true RIPC ( $n=12$ ) (inflating upper extremity blood pressure cuff thrice for 5 minutes to $30 \mathrm{~mm} \mathrm{Hg}$ above systolic blood pressure) or sham RIPC $(n=12)$ (inflating blood pressure cuff thrice for 5 minutes to $30 \mathrm{~mm} \mathrm{Hg}$ ) in a 1:1 allocation ratio using a computerized allocation sequence and block randomization. Our outcome measures, assessed by an observer blinded to the study intervention, were vasospasm on cerebral angiography and transcranial Doppler (TCD) study, and serum concentration of biomarkers of astrocytic and neuronal damage S100B and NSE at 24 hours after RIPC and on day 7 of ictus.

Results: Incidence of vasospasm on angiography ( $n=13$ ) was $25 \%$ (1/4 patients) in RIPC group and 89\% (8/9 patients) in sham group ( $p=0.052$ ). Vasospasm on TCD study was diagnosed in $1 / 13$ patients (7.7\%) and $4 / 12$ patients (33.3\%) in true and sham RIPC groups, respectively ( $p=0.16$ ). There was no difference in serum concentrations of S100B and NSE between groups ( $p=0.56$ and 0.31 , respectively ) and over time in either groups ( $p=0.318$ and 0.494 for S100B and $p=0.661$ and 0.174 for NSE in RIPC and sham groups, respectively). None had adverse effects on transient limb ischemia with RIPC.

Conclusions: This pilot trial showed that RIPC is feasible and safe. Our preliminary findings suggest a protective role of RIPC in prevention of vasospasm after aSAH, which needs confirmation by a larger trial.

A0023 Effect of Perioperative Hemoglobin Concentration on Cerebral Oxygen Extraction and Neurological Outcome following Aneurysmal Subarachnoid Hemorrhage

Deepak Rajappa, ${ }^{1}$ Hemant Bhagat, ${ }^{1}$ Nidhi Panda,

Ankur Luthra, ${ }^{1}$ Ashish Aggarwal ${ }^{2}$

${ }^{1}$ Department of Anaesthesia and Intensive Care, Postgraduate Institute of Medical Education and Research, Chandigarh, India ${ }^{2}$ Department of Neurosurgery, Postgraduate Institute of Medical Education and Research, Chandigarh, India

Background: The decrease in hemoglobin concentration ( $\mathrm{Hb}$ ) levels has a better rheological and cerebral perfusion spectrum. However, reduced Hb levels are associated with decreased cerebral oxygenation as a result of reduction in oxygen carrying capacity. On the contrary, higher Hb levels compromise blood rheology and cerebral perfusion. Optimal $\mathrm{Hb}$ in subarachnoid hemorrhage (SAH) patients is still controversial for better outcome of the SAH patients. The study aimed to evaluate the effect of perioperative $\mathrm{Hb}$ levels on cerebral oxygen extraction and neurological outcome in SAH patients.

Materials and Methods: Patients undergoing aneurysmal clipping for ruptured SAH were included. After anesthesia induction and following placement of jugular venous catheter, patients were additionally monitored for partial pressure of oxygen in arterial blood $\left(\mathrm{PaO}_{2}\right)$, saturation in arterial blood $\left(\mathrm{SaO}_{2}\right)$, saturation in jugular venous blood $\left.(\mathrm{SjvO})_{2}\right)$, and $\mathrm{Hb}$. Samples were obtained hourly in the intraoperative period and twice daily for 3 days in postoperative period to know $\mathrm{Hb}$ and cerebral oxygen extraction. Neurological outcome of the patients was measured with mRS and GOS-E at 7 days and 3 months, respectively. Hb values were correlated with arteriovenous difference in oxygenation $\left(\mathrm{AVDO}_{2}\right)$ and neurological outcome.

Results: Eighty patients were included in the study. Significant correlation was obtained between intraoperative and postoperative $\mathrm{Hb}$ values with GOS-E at 3 months $(p<0.05)$. No correlation was seen between $\mathrm{Hb}$ and MRS at 7 days. There was a positive linear relationship between $\mathrm{Hb}$ and $\mathrm{AVDO}_{2}$ values $(p<0.001)$. Multivariate analysis showed intraoperative $\mathrm{Hb}<10 \mathrm{~g} / \mathrm{dL}$ as an independent risk factor for poor neurological outcome.

Conclusions: Perioperative $\mathrm{Hb}$ values correlate with GOS-E at 3 months. Mean $\mathrm{Hb}$ of $<10 \mathrm{~g} / \mathrm{dL}$ during perioperative period appears to have an unfavorable neurological outcome at 3 months following aneurysmal SAH. 